Research Article

\title{
The Limit Theorems for Function of Markov Chains in the Environment of Single Infinite Markovian Systems
}

\author{
Zhanfeng Li, ${ }^{1}$ Min Huang, ${ }^{1,2}$ Xiaohua Meng, ${ }^{3}$ and Xiangyu Ge $\mathbb{D}^{1}$ \\ ${ }^{1}$ School of Statistics and Mathematics, Zhongnan University of Economics and Law, Wuhan 430073, China \\ ${ }^{2}$ Faculty of Information and Engineering, Wuhan College, Wuhan 430212, China \\ ${ }^{3}$ Institute of Information Management and Statistics, Hubei University of Economics, Wuhan 430205, China \\ Correspondence should be addressed to Xiangyu Ge; xiangyu_ge@163.com
}

Received 6 March 2020; Accepted 15 April 2020; Published 5 May 2020

Guest Editor: Wenguang Yu

Copyright ( $\odot 2020$ Zhanfeng Li et al. This is an open access article distributed under the Creative Commons Attribution License, which permits unrestricted use, distribution, and reproduction in any medium, provided the original work is properly cited.

This paper is intended to study the limit theorem of Markov chain function in the environment of single infinite Markovian systems. Moreover, the problem of the strong law of large numbers in the infinite environment is presented by means of constructing martingale differential sequence for the measurement under some different sufficient conditions. If the sequence of even functions $\left\{g_{n}(x), n \geq 0\right\}$ satisfies different conditions when the value ranges of $x$ are different, we have obtained SLLN for function of Markov chain in the environment of single infinite Markovian systems. In addition, the paper studies the strong convergence of the weighted sums of function for finite state Markov Chains in single infinitely Markovian environments. Although the similar conclusions have been carried out, the difference results performed by previous scholars are that we give weaker different sufficient conditions of the strong convergence of weighted sums compared with the previous conclusions.

\section{Introduction}

The definition and properties of limit theorems have been studied for some time, especially for functions of Markov chain, which becomes one of the most popular research areas in the field of stochastic processes. In a random process model, the theory of Markov chain describes the change from one system state to another system state, and also the advent of quantitative analysis is explored according to the real system situations. In effect, the theory of Markov chain is not only widely used in scientific research but also used in the economic field. Recently, emphasis is placed on the application to explain many systemic problems of economic phenomena, and the interpretation of most economic phenomena can be realized under the framework of Markov chain.

Over the course of the past 40 years, a comprehensive study of the Markov chains has been undertaken. In the early 1980s, Cogburn [1] introduced the definition of Markov chain in the environment of random systems and discussed the state classification of Markov chain in the environment of Markovian systems. A relevant paper was published by Nawrotzki [2,3], which discussed the state of classification about the Markov chain with feedback, based on other systems, namely, the single infinitely stable Markovian systems, and established the general theory of the topic. Subsequently, a further study on Markov chains under the condition of random environment has been reported by Cogburn [4] who have made a great contribution in this area. In random environments, Cogburn proposed a generic theory about the function of Markov chains and developed a lot of profound results with the theory of Hopf-Markov chain and some further studies of limit theories for function of Markov chain have been conducted. For instance, Cogburn $[5,6]$ discussed the convergence of the transfer probability, periodicity, and conditions for the establishment of the central limit theorem under the special circumstances, which are in the environment of the bi-infinite stable, as well as listing the connection with these theories. Orey [7] has studied the Markov chain in stochastic environment in depth based on Cogburn's study and put forward a series of problems, which attracted the attention of many 
probability scholars. Liu and Liu [8,9] have investigated a series of limit properties of the random variables sequences with Lebesgue's theorem and then gave limit properties with the similar method for nonhomogeneous Markov chain.

The general theory about the function of Markov chain in the environment of random systems has become a popular research direction. As known, the limit theorem has been a hot topic in the study of classical Markov chain theory. Subsequently, a lot of scholars have conducted indepth research in this field and achieved a series of profound and rich results. Various research theories about the function of Markov chain in Markovian environments have been proposed, called MCME for short (see [10-15]), and the same as theories about the function of Markov chain in random environments, which are called MCRE for short (see [16-18]). Exactly, the random environments can be catalogued into different situations, such as in space-time random environments (see [19]), in bi-infinite random environments (see [20]), and in single infinite random environments (see [21]).

Currently, a lot of research literatures on the strong limit theory for function of Markov chains in the environment of random systems or in the environment of Markovian systems have been found. The strong law of large numbers about the function of Markov chains in the environment of Markovian systems with discrete parameter was proposed by Wan [22] who obtained the sufficient conditions. Besides, Guo [16] also put forward the sufficient conditions, which are different from Wan [22], and the difference is mainly to prove this theorem in the case of the random environments. On the basis of existing research, $\mathrm{Li}$ [23] indicated the sufficient conditions established in the case of countable states for this theorem. Furthermore, for complete and imprecise knowledge of Markov chains, Li et al. [14] have developed a strong limit theorem of the Markov chain quaternion function in the environment of Markovian systems and extended the Shannon theorem in this environment. It becomes apparent that Markovian environments can be classified into different catalogues, such as in bi-infinite environments (see $[24,25])$ and in single infinite environments (see [26]).

To the best of our knowledge, along with the increasing development of Markov chains in decision-making state of financial market, they have been widely used in financial insurance theory. Recently, statistical estimation of ruin related functions has become a popular topic in risk theory. However, some of the scholars proposed different estimators for the ruin probability in the classical risk. For example, a study by Yang and Yuen [27] offered a comprehensive analysis of two-dimensional delayed renewal risk model with a constant interest. They derived some asymptotic formulas for the finite-time and infinite-time ruin probabilities in the presence of heavy-tailed claim sizes. In addition, Yang et al. [28] constructed by the two-dimensional Fourier cosine series expansion to estimate the discounted density of the deficit at ruin. Similarly, with method of the Fourier cosine series expansion, one study by $\mathrm{Yu}$ et al. [29] valued the guaranteed minimum death benefit products. On valuation of the products with guaranteed minimum death benefit,
Zhang et al. [30] applied a projection method combined with Fast Fourier Transform. Recently, a qualitative study by Yang et al. [31] described a discrete-time insurance risk model with insurance and financial risks. Then, a key study is that of Yu et al. [32], which proposed a new risk model called compound Poisson risk model by introducing a periodic capital injection strategy and a barrier dividend strategy into the classical risk model. Furthermore, the risk model can be further extended and applied to a wider range of practical problems. An example can be made by the optimal control problem (see [33]). Above all, most of existing studies failed to deal with the practical problems; it is necessary for us to study the limit theorems for function of Markov chains deeply.

Therefore, this paper set out to advance the research on the limit theory of a class of Markov chain functions, aiming to provide clarity understanding of the limit theorem for function of Markov chain in environment of single infinite Markovian systems. Based on the results of Li [17] and Wan [18], we have found two lemmas and derive the results of almost sure convergence with the finding of the lemmas. The results of the analysis of the strong law of large numbers in single infinite environment are presented to be an extension of the conclusion in the inference [34] on different sufficient conditions. Also, in single infinite Markovian environments, we come to the conclusions about the strong convergence and present the weighted sums for function of Markov chains. Driven by LLN (law of large numbers), the strong convergence of the weighted sum is also discussed when considering the compatibility of the least squares estimates of linear models. Although we prove similar conclusions, the difference from results obtained by previous scholars can be made based on weaker different sufficient conditions of the strong convergence of weighted sums provided [26].

The subsequent structure of our paper is as follows. A description of some basic notations, fundamental definitions, and lemmas is shown briefly in Section 2. Details under the condition of the environment of single infinite Markovian systems are discussed in Section 3 and Section 4. Section 3 is about SLLN (strong law of large numbers) for function of Markov chain in the environment of the setting of this paper. Section 4 derives the strong convergence of weighted sum for function of Markov chain in the environment of the setting of this paper. A series of sufficient conditions for the limit theorem are obtained in Sections 3 and 4 , and specific proof process of theorem and corollary is considered, respectively. This paper concludes with a discussion in Section 5.

\section{Fundamental Preliminaries}

At first, we begin to introduce some basic notations which shall be used in the following sections. Let $N$ represent an integer set and $(\Omega, \mathscr{F}, P)$ represent a probability space; both $(X, \mathscr{A})$ and $(\Theta, \mathscr{B})$ are arbitrary measurable spaces. Respectively, let $\vec{\xi}_{0}^{\infty}=\left\{\xi_{n}: n \geq 0\right\}$ and $\vec{X}=\left\{X_{n}: n \geq 0\right\}$ be two 
random sequences defined on $(\Omega, \mathscr{F}, P)$ with value on set of $\Theta$ and $X$. Assume a family of transition function $\{P(\theta): \theta \in \Theta\}$ defined on arbitrary measurable spaces of $(X, \mathscr{A})$. For any $A \in \mathscr{A}$, we suppose $P(\cdot ; \cdot, A)$ is measurable regarding $\mathscr{B} \times \mathscr{A}$. Given a family of one-step transition probability function $\left\{K_{n}(\cdot, \cdot)\right\}$ defined on arbitrary measurable spaces of $(\Theta, \mathscr{B})$, where we assume that $K_{n}(\cdot, B)$ is measurable about $\mathscr{B}$ for any $B \in \mathscr{B}$. For arbitrary sequence of $\vec{\eta}=\left\{\eta_{n}: n \geq 0\right\}$, we denote $\vec{\eta}_{k}^{r}=\left\{\eta_{n}: 0 \leq n \leq r \leq \infty\right\}$.

If, for any of $A \in \mathscr{A}$ and $n \geq 0$, at the same time we have

$$
\begin{aligned}
P\left(X_{0} \in A \mid \vec{\xi}_{0}^{\infty}\right) & =P\left(X_{0} \in A \mid \xi_{0}\right), \\
P\left(X_{n+1} \in A \mid \vec{X}_{0}^{n}, \vec{\xi}_{0}^{\infty}\right) & =P\left(\xi_{n} ; X_{n}, A\right),
\end{aligned}
$$

then random variable sequence of $\vec{X}$ is called the Markov chain in the random system of $\vec{\xi}_{0}^{\infty}$; here $\vec{\xi}_{0}^{\infty}$ is a sequence in single infinite random environments. In other words, if $\vec{\xi}_{0}^{\infty}$ is a Markov sequence, $\vec{X}$ is called the Markov chain in the environment of single infinite Markovian systems.

Given a random variable sequence $\left\{X_{n}, n \geq 0\right\}$ on $(\Omega, \mathscr{F}, P)$, the following statement is satisfied. If, for arbitrary $x>0$ and $n \geq 0$, there exists $P\left(\left|X_{n}\right|>x\right) \leq C P(V>x)$, where $V$ represents a nonnegative random variable, $C$ appearing here represents a constant and is greater than zero. Thus we call $\left\{X_{n}, n \geq 0\right\}$ the tail probability uniformly bounded by $V$ and denote it as $\left\{X_{n}\right\}<V$. This paper always sets $\vec{X}$ as the Markov chain in single infinite Markovian environments. We assume that $C$ appearing in this paper represents a positive constant that represents different values in different positions. The indicative function of the set of $A$ is denoted as $I_{A}$.

The purpose of the study is to propose limit theorems for function of Markov chain in analysis of the environment of single infinite Markovian systems and to conclude some different sufficient conditions for the almost sure convergence by means of constructing martingale differential sequence. In addition, we derive the similar conclusion about strong convergence of the weighted sums in the given environment in terms of some weaker sufficient conditions.

Two specific contributions have been included as follows, which are both built on the environment of single infinite Markovian systems. Firstly, this study will offer a fresh insight into the following Theorem 1 and Theorem 2 and Corollaries 1 and 2 to show Markov chain's SLLN (strong law of large numbers) in the given environment. Secondly, this study will provide an important opportunity to advance the understanding of Markov chain strong convergence of weighted sum in the given environment. The results are given by Theorem 3 and Corollary 3 .

To prove the main theorems, the following deformation lemmas are needed.

Lemma 1 (see, e.g., Conclusion 1 in [17]). Given a Markov chain $\vec{X}$ in the environment of single infinite Markovian systems $\vec{\xi}_{0}$, the random variable sequence of $\left\{\left(X_{n}, \xi_{n}\right): n \geq 0\right\}$ is the Markovian chain in double. In particular, if the one-step transition function in the single infinite Markovian environments $\vec{\xi}_{0}^{\infty}$ is $K_{n}(\theta, B)$, the onestep transition probability of $\left\{\left(X_{n}, \xi_{n}\right): n \geq 0\right\}$ is

$$
Q_{n}(x, \theta ; A \times B)=K_{n}(\theta, B) P(\theta ; x, A) .
$$

If $\vec{\xi}_{0}^{\infty}$ is time-homogeneous, then $\left\{\left(X_{n}, \xi_{n}\right): n \geq 0\right\}$ is time-homogeneous too.

Lemma 2 (see Lemma 2 in [18]). Assume that $X$ is a random variable; for any $x>0$, there exists $P(|X|>x) \leq C P(V>x)$, where $V$ is a nonnegative random variable and $C>0$ is a constant, such that, for any $x>0$ and $q>0$, we have

$$
E|X|^{q} I_{\{|X| \leq x\}} \leq C x^{q} P(V>x)+C E V^{q} I_{\{V \leq x\}} .
$$

\section{SLLN in the Environment of Single Infinite Markovian Systems}

To begin with, we use Lemmas 1 and 2 to investigate SLLN for function of Markov chain in the environment of single infinite Markovian systems. A series of sufficient conditions for SLLN are given for function of Markov Chains in the environment of single infinite Markovian systems. The relevant results are in accordance with the following major Theorems 1 and 2, as well as Corollaries 1 and 2 .

Theorem 1. Given the probability space of $(\Omega, \mathscr{F}, P)$ with values on set of $X \times \Theta$, we assume that $\left\{\left(X_{n}, \xi_{n}\right): n \geq 0\right\}$ is a Markov chain, $\left\{F_{n}\left(X_{n}, \xi_{n}\right): n \geq 0\right\}$ is a sequence of measurable functions defined on $(X \times \Theta, \mathscr{A} \times \mathscr{B})$, and $\left\{g_{n}(x), n \geq 0\right\}$ is a sequence of even functions defined on set of $R$ which is taking a positive value on the interval of $(0, \infty)$. For any $n \geq 0$, there always exists a value of $\lambda>0$, if one of the following conditions holds:

(i) $g_{n}(x)$ is monotonically nondecreasing on the interval of $(0, \infty)$. When $0<x \leq 1$, there is $g_{n}(x) \geq \lambda x^{\theta}$ $(0<\theta \leq 1)$, and $E\left(F_{n}\left(X_{n}, \xi_{n}\right)\right)=0, \quad n \geq 0$;

(ii) $g_{n}(x) \geq \begin{cases}\lambda x^{\alpha}(0<\alpha \leq 2), & 0<x \leq 1, \\ \lambda x^{\beta}(\beta \geq 1), & x>1 .\end{cases}$

At the same time, for positive constant sequences of $\left\{a_{n}, n \geq 0\right\}$ where $a_{n} \uparrow \infty$ is satisfied, if there exist

$$
\sum_{m=0}^{\infty} E g_{m}\left(\frac{F_{m}\left(X_{m}, \xi_{m}\right)}{a_{m}}\right)<\infty,
$$

then, for any $k \geq 1$, we get the following series, which are convergent almost surely:

$$
\begin{aligned}
& \sum_{m=0}^{\infty} \frac{F_{m}\left(X_{m}, \xi_{m}\right)-E\left(F_{m}\left(X_{m}, \xi_{m}\right) \mid X_{m-k}, \xi_{m-k}\right)}{a_{m}} \\
& \text { ·a.s.convergence, }
\end{aligned}
$$

and consequently we have the following formula, which is true almost surely: 


$$
\begin{aligned}
& \lim _{n \longrightarrow \infty} \frac{1}{a_{n}} \sum_{m=0}^{n}\left(F_{m}\left(X_{m}, \xi_{m}\right)\right. \\
& \left.-E\left(F_{m}\left(X_{m}, \xi_{m}\right) \mid X_{m-k}, \xi_{m-k}\right)\right)=0 \text { a.s.. }
\end{aligned}
$$

Here, we agree $X_{-k} \equiv 0$ and $\xi_{-k} \equiv 0$ for any $k \geq 1$.

According to the derivation of Theorem 1, we can further generalize Corollary 1 as follows.

Corollary 1. Assume a Markov chain $\left\{\left(X_{n}, \xi_{n}\right): n \geq 0\right\}$ which is defined on probability space of $(\Omega, \mathscr{F}, P)$ with values on set of $X \times \Theta .\left\{F_{n}\left(X_{n}, \xi_{n}\right): n \geq 0\right\}$ is a sequence of measurable functions defined on $(X \times \Theta, \mathscr{A} \times \mathscr{B})$. For the positive constant sequences of $\left\{a_{n}, n \geq 0\right\}$, satisfying $a_{n} \uparrow \infty$, if one of the following two conditions holds:

(iii) $\sum_{m=0}^{\infty} E\left(\left|F_{m}\left(X_{m}, \xi_{m}\right)\right|^{r} /\left(\left|a_{m}\right|^{r}+\left|F_{m}\left(X_{m}, \xi_{m}\right)\right|^{r}\right)\right)$ $<\infty$, where $0<r<1$ and $E\left(F_{n}\left(X_{n}, \xi_{n}\right)\right)=0, n \geq 0$; (iv) $\sum_{m=0}^{\infty} E\left(\left(\left|F_{m}\left(X_{m}, \xi_{m}\right)\right|^{r}\right) /\left(\left|a_{m}\right|^{r}+a_{m} \mid F_{m}\left(X_{m}\right.\right.\right.$, $\left.\left.\left.\xi_{m}\right)\left.\right|^{r-1}\right)\right)<\infty$, where $1 \leq r \leq 2$, then (5) and (6) hold.

Proof of Theorem 1. Let us now prove the above Theorem 1. In the process of proof, we discuss two situations. One situation is $k=1$, and the other situation is $k>1$.

Firstly, we consider the situation of $k=1$.

Under condition (i), there satisfies $\left|F_{n}\left(X_{n}, \xi_{n}\right)\right|>a_{n}$, that is, $\left(\left|F_{n}\left(X_{n}, \xi_{n}\right)\right| / a_{n}\right)>1$. We know from the condition that $g_{n}(x)$ is monotonically nondecreasing on the interval of $(0, \infty)$, and we can get $g_{n}(1) \geq \lambda$ when condition (i) is satisfied. Thus, we can get the inequality $g_{n}\left(\left|F_{n}\left(X_{n}, \xi_{n}\right)\right| / a_{n}\right) \geq C g_{n}(1)$ which will be used in the following derivation. Next, let us give the detailed process of the derivation of inequalities (7) and (8) by using Lemma 2. The derivation of inequality is as follows:

$$
\begin{aligned}
& \sum_{m=0}^{\infty} P\left(\left|F_{m}\left(X_{m}, \xi_{m}\right)\right|>a_{m}\right) \leq C \sum_{m=1}^{\infty} E g_{m}(1) I_{\left\{\left|F_{m}\left(X_{m}, \xi_{m}\right)\right|>a_{m}\right\}} \\
& \leq C \sum_{m=1}^{\infty} E g_{m}\left(\frac{\left|F_{m}\left(X_{m}, \xi_{m}\right)\right|}{a_{m}}\right) I_{\left\{\left|F_{m}\left(X_{m}, \xi_{m}\right)\right|>a_{m}\right\}} \\
& \leq C \sum_{m=1}^{\infty} E g_{m}\left(\frac{\left|F_{m}\left(X_{m}, \xi_{m}\right)\right|}{a_{m}}\right)<\infty, \\
& E(\left.\sum_{m=0}^{\infty}\left|E\left(\frac{F_{m}\left(X_{m}, \xi_{m}\right)}{\left.a_{m} I_{\left\{\mid F_{m}\right.}\left(X_{m}, \xi_{m}\right) \mid>a_{m}\right\}} \mid X_{m-1}, \xi_{m-1}\right)\right|\right) \\
&=E\left(\sum_{m=0}^{\infty}\left|E\left(\frac{F_{m}\left(X_{m}, \xi_{m}\right)}{a_{m}} I_{\left\{\left|F_{m}\left(X_{m}, \xi_{m}\right)\right| \leq a_{m}\right\}} \mid X_{m-1}, \xi_{m-1}\right)\right|\right) \\
&\left.\leq \sum_{m=0}^{\infty} E\left(\frac{\left|F_{m}\left(X_{m}, \xi_{m}\right)\right|}{a_{m}} I_{\left\{\mid F_{m}\right.}\left(X_{m}, \xi_{m}\right) \mid \leq a_{m}\right\}\right) \\
&\left.\leq \sum_{m=0}^{\infty} E\left(\frac{\left|F_{m}\left(X_{m}, \xi_{m}\right)\right|}{a_{m}^{\theta}}\right) I_{\left\{\left|F_{m}\left(X_{m}, \xi_{m}\right)\right| \leq a_{m}\right\}}\right) \\
& \leq C \sum_{m=0}^{\infty} E \sum_{m=0}^{\infty} E g_{m}\left(\frac{\left|F_{m}\left(X_{m}, \xi_{m}\right)\right|}{a_{m}\left(X_{m}, \xi_{m}\right) \mid} a_{m}\right)<\infty . \\
&\left\{\left|F_{m}\left(X_{m}, \xi_{m}\right)\right| \leq a_{m}\right\} \\
&
\end{aligned}
$$


Under condition (ii), when $\left|F_{n}\left(X_{n}, \xi_{n}\right)\right|>a_{n}$ is satisfied at the same time, we use the second case of condition (ii) $g_{n}(x) \geq \lambda x^{\beta}(\beta \geq 1, x>1)$ to get the inequality $g_{n}\left(\left|F_{n}\left(X_{n}, \xi_{n}\right)\right| / a_{n}\right) \geq C\left(\left|F_{n}\left(X_{n}, \xi_{n}\right)\right|^{\beta} / a_{n}^{\beta}\right)$ which will be used in the following derivation. Next, let us give the detailed process of the derivation of inequalities (9) and (10). The derivation of inequality is as follows:

$$
\begin{gathered}
\sum_{m=0}^{\infty} P\left(\left|F_{m}\left(X_{m}, \xi_{m}\right)\right|>a_{m}\right) \leq C \sum_{m=0}^{\infty} E\left(\frac{\left|F_{m}\left(X_{m}, \xi_{m}\right)\right|^{\beta}}{a_{m}^{\beta}}\right) I_{\left\{\left|F_{m}\left(X_{m}, \xi_{m}\right)\right|>a_{m}\right\}} \\
\leq C \sum_{m=0}^{\infty} E g_{m}\left(\frac{\left|F_{m}\left(X_{m}, \xi_{m}\right)\right|}{a_{m}}\right)<\infty \\
E\left(\sum_{m=0}^{\infty}\left|E\left(\frac{F_{m}\left(X_{m}, \xi_{m}\right)}{a_{m}} I_{\left\{\left|F_{m}\left(X_{m}, \xi_{m}\right)\right|>a_{m}\right\}} \mid X_{m-1}, \xi_{m-1}\right)\right|\right) \\
\leq \sum_{m=0}^{\infty} E\left(\frac{\left|F_{m}\left(X_{m}, \xi_{m}\right)\right|}{a_{m}} I_{\left\{\left|F_{m}\left(X_{m}, \xi_{m}\right)\right|>a_{m}\right\}}\right) \\
\leq \sum_{m=0}^{\infty} E\left(\frac{\left|F_{m}\left(X_{m}, \xi_{m}\right)\right|^{\beta}}{a_{m}^{\beta}}\right) I_{\left\{\left|F_{m}\left(X_{m}, \xi_{m}\right)\right|>a_{m}\right\}} \\
\leq C \sum_{m=0}^{\infty} E g_{m}\left(\frac{\left|F_{m}\left(X_{m}, \xi_{m}\right)\right|}{a_{m}}\right)<\infty
\end{gathered}
$$

Obviously, by using formulas (7) and (9), it is obvious that the result below is established almost surely:

$$
\sum_{m=0}^{\infty} I_{\left\{\left|F_{m}\left(X_{m}, \xi_{m}\right)\right|>a_{m}\right\}}<\infty \text { a.s.. }
$$

Because $P\left(\left|F_{m}\left(X_{m}, \xi_{m}\right)\right|>a_{m}\right.$ : i.o. $)=0$, consequently we can get that the following series are convergent almost surely:

$$
\sum_{m=0}^{\infty} \frac{F_{m}\left(X_{m}, \xi_{m}\right)}{a_{m}} I_{\left\{\left|F_{m}\left(X_{m}, \xi_{m}\right)\right|>a_{m}\right\}} \text { a.s.convergence. }
$$

At the same time, by using formulas (8) and (10), we can get that the following series are convergent almost surely:

$$
\sum_{m=0}^{\infty} E\left(\frac{F_{m}\left(X_{m}, \xi_{m}\right)}{a_{m}} I_{\left\{\left|F_{m}\left(X_{m}, \xi_{m}\right)\right|>a_{m}\right\}} \mid X_{m-1}, \xi_{m-1}\right) \text { a.s.convergence. }
$$

Note that

$$
\begin{aligned}
Z_{n}= & \frac{F_{n}\left(X_{n}, \xi_{n}\right) I_{\left\{\left|F_{n}\left(X_{n}, \xi_{n}\right)\right| \leq a_{n}\right\}}}{a_{n}} \\
& -\frac{E\left(F_{n}\left(X_{n}, \xi_{n}\right) I_{\left\{\left|F_{n}\left(X_{n}, \xi_{n}\right)\right| \leq a_{n}\right\}} \mid X_{n-1}, \xi_{n-1}\right)}{a_{n}}, \\
\mathscr{B}_{n}= & \sigma\left(\vec{X}_{0}^{n}, \vec{\xi}_{0}^{n}\right) .
\end{aligned}
$$

$\left\{Z_{n}, \mathscr{B}_{n}, n \geq 0\right\}$ is known as a martingale difference sequence by the nature of function of Markov chain of $\left\{\left(X_{n}, \xi_{n}\right), n \geq 0\right\}$. Under condition (i), the following results can be derived by the orthogonality of martingale difference sequence:

$$
\begin{aligned}
E\left|\sum_{m=0}^{n} Z_{m}\right|^{2} & =\sum_{m=0}^{n} E Z_{m}^{2} \\
& \leq C \sum_{m=0}^{n} E\left(\frac{\left|F_{m}\left(X_{m}, \xi_{m}\right)\right|^{2}}{a_{m}^{2}} I_{\left\{\left|F_{m}\left(X_{m}, \xi_{m}\right)\right| \leq a_{m}\right\}}\right) \\
& \leq C \sum_{m=0}^{n} E\left(\frac{\left|F_{m}\left(X_{m}, \xi_{m}\right)\right|^{\theta}}{a_{m}^{\theta}} I_{\left\{\left|F_{m}\left(X_{m}, \xi_{m}\right)\right| \leq a_{m}\right\}}\right) \\
& \leq C \sum_{m=0}^{\infty} E g_{m}\left(\frac{\left|F_{m}\left(X_{m}, \xi_{m}\right)\right|}{a_{m}}\right) I_{\left\{\left|F_{m}\left(X_{m}, \xi_{m}\right)\right| \leq a_{m}\right\}} \\
& \leq C \sum_{m=0}^{\infty} E g_{m}\left(\frac{\left|F_{m}\left(X_{m}, \xi_{m}\right)\right|}{a_{m}}\right) .
\end{aligned}
$$

Also, under condition (ii), the inequality is derived as follows:

$$
\begin{array}{r}
E\left|\sum_{m=0}^{n} Z_{m}\right|^{2}=\sum_{m=0}^{n} E Z_{m}^{2} \\
\leq C \sum_{m=0}^{n} E\left(\frac{\left|F_{m}\left(X_{m}, \xi_{m}\right)\right|^{2}}{a_{m}^{2}} I_{\left\{\left|F_{m}\left(X_{m}, \xi_{m}\right)\right| \leq a_{m}\right\}}\right) \\
\leq C \sum_{m=0}^{n} E\left(\frac{\left|F_{m}\left(X_{m}, \xi_{m}\right)\right|^{\alpha}}{a_{m}^{\alpha}} I_{\left\{\left|F_{m}\left(X_{m}, \xi_{m}\right)\right| \leq a_{m}\right\}}\right) \\
\leq C \sum_{m=0}^{\infty} E g_{m}\left(\frac{\left|F_{m}\left(X_{m}, \xi_{m}\right)\right|}{a_{m}}\right) .
\end{array}
$$

By formula (4), we know that $\sup _{n \geq 0} E\left|\sum_{m=0}^{n} Z_{m}\right|^{2}<\infty$, which means $\left\{\sum_{m=0}^{n} Z_{m}, \mathscr{B}_{n}, n \geq 0\right\}$ is bounded martingale on 
$L^{2}$. Thus, we can get that the series $\sum_{m=0}^{\infty} Z_{m}$ are convergent almost surely. Then, by combining formulas (12) and (13), we can see that formula (5) holds, and it is also easy to know that formula (6) holds by Kronecker's Lemma.

Secondly, we consider the other situation of $k>1$.

By the nature of function of the Markov chain $\left\{\left(X_{n}, \xi_{n}\right): n \geq 0\right\}$, it is known that $\left\{\left(X_{m k+n}, Y_{m k+n}\right): m \geq 0\right\}$ is a Markov chain for any $n=1,2,3, \ldots, k-1$. It is easy to derive the following result regarding convergence by using formula (4):

$$
\sum_{m=0}^{\infty} E g_{m k+n}\left(\frac{F_{m k+n}\left(X_{m k+n}, \xi_{m k+n}\right)}{a_{m k+n}}\right)<\infty
$$

Therefore, for any $n=1,2,3, \ldots, k-1$, we have the series of almost sure convergence as follows:

$$
\sum_{m=0}^{\infty} \frac{F_{m k+n}\left(X_{m k+n}, \xi_{m k+n}\right)-E\left(F_{m k+n}\left(X_{m k+n}, \xi_{m k+n}\right) \mid X_{m k+n-k}, \xi_{m k+n-k}\right)}{a_{m k+n}} \text { a.s.convergence. }
$$

Thus, the following results of convergence almost surely can be derived:

$$
\begin{aligned}
\sum_{m=0}^{\infty} & \frac{F_{m}\left(X_{m}, \xi_{m}\right)-E\left(F_{m}\left(X_{m}, \xi_{m}\right) \mid X_{m-k}, Y_{m-k}\right)}{a_{m}} \\
& =\sum_{m=0}^{\infty} \sum_{n=0}^{k-1} \frac{F_{m k+n}\left(X_{m k+n}, \xi_{m k+n}\right)-E\left(F_{m k+n}\left(X_{m k+n}, \xi_{m k+n}\right) \mid X_{m k+n-k}, \xi_{m k+n-k}\right)}{a_{m k+n}} \\
& =\sum_{n=0}^{k-1} \sum_{m=0}^{\infty} \frac{F_{m k+n}\left(X_{m k+n}, \xi_{m k+n}\right)-E\left(F_{m k+n}\left(X_{m k+n}, \xi_{m k+n}\right) \mid X_{m k+n-k}, \xi_{m k+n-k}\right)}{a_{m k+n}},
\end{aligned}
$$

where, for the situation of $k>1$, formula (6) is true. Obviously, by Kronecker's Lemma, formula (7) also holds for the situation of $k>1$.

Now, the conclusions on convergence have been demonstrated and proven completely. The method of using constructing martingale differential sequence to implement different sufficient conditions of the strong limit theorems is different from the evidence from previous observations. Our paper advances the research on the limit theory of a class of Markov chain functions by using different sufficient conditions.

Remark 1. Both Theorem 1 and Lemma 4 in literature [24] give the sufficient conditions for SLLN of Markov chain, but the preconditions in the two theorems are different.

From Theorem 1, we can see that the sequence of even functions $\left\{g_{n}(x), n \geq 0\right\}$ satisfies different conditions when the value ranges of $x$ are different. In condition (i), when $0<x \leq 1$ and $0<\theta \leq 1, g_{n}(x)$ needs to be monotonically nondecreasing and $E\left(F_{n}\left(X_{n}, \xi_{n}\right)\right)=0$ with $n \geq 0$. However, in condition (ii), these conditions are not required to approach limit but only to obtain the segment $g_{n}(x)$ and also a range of values are satisfied. From these two different sufficient conditions, we have obtained SLLN for function of Markov chain in the environment of single infinite Markovian systems. On the basis of Theorem 1, Corollary 1 shows that SLLN can be obtained by assigning different functional forms to $g_{n}(x)$, which generalize the previous conclusions.
Proof of Corollary 1. At first, when condition (iii) is established, there exists $g_{n}(x)=|x|^{r} /\left(1+|x|^{r}\right)$, where the range of value of $r$ is $0<r<1$. When condition (iv) is established, there is $g_{n}^{\prime}(x)=|x|^{r} /\left(1+|x|^{r-1}\right)$, where the range of value of $r$ is $1 \leq r \leq 2$. Then, for any $n \geq 0, g_{n}(x)$ and $g_{n}^{\prime}(x)$ are both even nondecreasing functions, taking a positive value on interval of $(0, \infty)$. At the same time, respectively, there are the following formulas:

$$
\begin{aligned}
& g_{n}(x) \geq \frac{1}{2} x^{r}, \quad 0<x \leq 1,0<r<1, \\
& g_{n}^{\prime}(x) \geq \begin{cases}\frac{1}{2} x^{r}, & 0<x \leq 1, \quad 1 \leq r \leq 2, \\
\frac{1}{2} x, & x>1 .\end{cases}
\end{aligned}
$$

If condition (iii) is satisfied, we have

$\sum_{m=0}^{\infty} E g_{n}\left(\frac{F_{m}\left(X_{m}, \xi_{m}\right)}{a_{m}}\right)=\sum_{m=0}^{\infty} E\left(\frac{\left|F_{m}\left(X_{m}, \xi_{m}\right)\right|^{r}}{\left|a_{m}\right|^{r}+\left|F_{m}\left(X_{m}, \xi_{m}\right)\right|^{r}}\right)<\infty$

If condition (iv) is satisfied, we have

$$
\sum_{m=0}^{\infty} E g_{m}^{\prime}\left(\frac{F_{m}\left(X_{m}, \xi_{m}\right)}{a_{m}}\right)=\sum_{m=0}^{\infty} E\left(\frac{\left|F_{m}\left(X_{m}, \xi_{m}\right)\right|^{r}}{\left|a_{m}\right|^{r}+a_{m}\left|F_{m}\left(X_{m}, \xi_{m}\right)\right|^{r-1}}\right)<\infty \text {. }
$$

From the above results, it can be seen that both condition (i) and condition (ii) under Theorem 1 are satisfied, so we can obtain that Corollary 1 holds by Theorem 1 . 
The sample frequency as a significant part of information theory plays a very important role in statistical hypothesis testing and coding theory. Based on the above results, a class of SLLN on the frequency of occurrence of the state are obtained for function of Markov chain in the environment of single infinite Markovian systems. Meanwhile, the relationship between the frequency of occurrence of state $(x, \theta)$ and the product of corresponding one-step transition probability function can be seen from the following Theorem 2. In particular, we can use the initial state to describe the frequency of occurrence of the state when the time-homogeneity is satisfied. Under the certain environment of the single infinite Markovian systems, we have concluded Corollary 2, which can be extended to a class of SLLN on the frequency of occurrence of the state for function of Markov chain.

Theorem 2. Assume that a Markov chain $X$ is in the environment of the single infinite Markovian systems $\overrightarrow{\xi_{0}^{\infty}}$. $S_{n}(x, \theta)$ represents the frequency where $(x, \theta)$ appears in the sequence of $\left(X_{1}, \xi_{1}\right),\left(X_{2}, \xi_{2}\right), \ldots,\left(X_{n}, \xi_{n}\right)$; if $\sum_{m=0}^{\infty} a_{m}^{-r}<\infty$, when $1<r \leq 2$, we then have

$$
\lim _{n \rightarrow \infty}\left(\frac{S_{n}(x, \theta)}{a_{n}}-\frac{1}{a_{n}} \sum_{m=0}^{n} K_{m}\left(\xi_{m-1}, \theta\right) P\left(\xi_{m-1} ; X_{m-1}, x\right)\right)=0 \text { a.s.. }
$$

In particular, when $\overrightarrow{\xi_{0}^{\infty}}$ is time-homogeneous and $\lim _{n \longrightarrow \infty}\left(n / a_{n}\right)=C$, then we have

$$
\lim _{n \longrightarrow \infty} \frac{S_{n}(x, \theta)}{a_{n}}=C K\left(\xi_{0}, \theta\right) P\left(\xi_{0} ; X_{0}, x\right) \text { a.s.. }
$$

Corollary 2. Assume that a Markov chain $X$ is in the environment of the single infinite Markovian systems $\overrightarrow{\xi_{0}^{\infty}} . S_{n}(x)$ represents the frequency where $x$ appears in the sequence of $X_{1}, X_{2}, \ldots, X_{n} ;$ if $\sum_{m=0}^{\infty} a_{m}^{-r}<\infty$, when $1<r \leq 2$, then we have

$$
\lim _{n \longrightarrow \infty}\left(\frac{S_{n}(x)}{a_{n}}-\frac{1}{n} \sum_{m=0}^{n} P\left(\xi_{m-1} ; X_{m-1}, x\right)\right)=0 \text { a.s.. }
$$

In particular, when $\overrightarrow{\xi_{0}^{\infty}}$ is time-homogeneous and $\lim _{n \longrightarrow \infty}\left(n / a_{n}\right)=C$, we have

$$
\lim _{n \longrightarrow \infty} \frac{S_{n}(x)}{a_{n}}=C P\left(\xi_{0} ; X_{0}, x\right) \text { a.s.. }
$$

Proof of Theorem 2. Given $F_{n}\left(X_{n}, \xi_{n}\right)=\delta_{x}\left(X_{n}\right) \delta_{\theta}\left(\xi_{n}\right)$, we have $S_{n}(x, \theta)=\sum_{m=1}^{n} F_{m}\left(X_{m}, \xi_{m}\right)$. Then the following inequality can be derived through equation (10) above:

$$
\sum_{m=0}^{\infty} E \frac{\left|F_{m}\left(X_{m}, \xi_{m}\right)\right|^{r}}{a_{m}\left|F_{m}\left(X_{m}, \xi_{m}\right)\right|^{r-1}+a_{m}^{r}} \leq \sum_{m=0}^{\infty} a_{m}^{-r}<\infty .
$$

So we can get from the above formula that the series is convergent.

Obviously, it is known that the following limit holds almost surely by Corollary 1 :

$$
\lim _{n \longrightarrow \infty} \frac{1}{a_{n}} \sum_{m=0}^{n}\left(F_{m}\left(X_{m}, \xi_{m}\right)-E\left(F_{m}\left(X_{m}, \xi_{m}\right) \mid X_{m-1}, \xi_{m-1}\right)\right)=0 \text { a.s.. }
$$

Then, by the combination of the formula $\sum_{m=0}^{n} F_{m}\left(X_{m}, \xi_{m}\right)=S_{n}(x, \theta)+\delta_{x}\left(X_{0}\right) \delta_{\theta}\left(\xi_{0}\right)$ and Lemma 1 , we can get the results below.

$$
\begin{aligned}
E\left(F_{n}\left(X_{n}, \xi_{n}\right) \mid X_{n-1}, \xi_{n-1}\right) & =E\left(\delta_{x}\left(X_{n}\right) \delta_{\theta}\left(\xi_{n}\right) \mid X_{n-1}, \xi_{n-1}\right) \\
& =Q_{n}\left(X_{n-1}, \xi_{n-1} ; x, \theta\right) \\
& =K_{n}\left(\xi_{n-1}, \theta\right) P\left(\xi_{n-1} ; X_{n-1}, x\right) .
\end{aligned}
$$

Substituting the above formula into the formula limitation equation (28), we can get formula (23).

If $\overrightarrow{\xi_{0}^{\infty}}$ is time-homogeneous, it is known that $\left\{\left(X_{n}, \xi_{n}\right): n \geq 0\right\}$ is also time-homogeneous by Lemma 1 , and hence $Q_{n}\left(X_{n-1}, \xi_{n-1} ; x, \theta\right)=Q\left(X_{0}, \xi_{0} ; x, \theta\right)=K\left(\xi_{0}, \theta\right) P\left(\xi_{0}\right.$; $\left.X_{0}, x\right)$. So we can get formula (24) by using formula (23). Therefore, Theorem 2 has been proven.

Proof of Corollary 2. Given the formula $F_{n}\left(X_{n}, \xi_{n}\right)=\delta_{x}\left(X_{n}\right)$, we have the formula $S_{n}(x)+\delta_{x}\left(X_{0}\right)=\sum_{m=0}^{n} F_{m}\left(X_{m}, \xi_{m}\right)=\sum_{m=0}^{n} \delta_{x}\left(X_{m}\right)$. By combining these conditions and Lemma 1 , we obtain the following results:

$$
E\left(F_{n}\left(X_{n}, \xi_{n}\right) \mid X_{n-1}, \xi_{n-1}\right)=E\left(\delta_{x}\left(X_{m}\right) \mid X_{n-1}, \xi_{n-1}\right)=P\left(\xi_{n-1} ; X_{n-1}, x\right),
$$

and, therefore, Corollary 2 can be proven by using the method which is similar to that used in the Proof of Theorem 2.

\section{The Strong Convergence of Weighted Sums in the Environment of Single Infinite Markovian Systems}

In this section, we use Lemmas 1 and 2 to study the strong convergence of weighted sum for the function of Markov chain in the environment of the single infinite Markovian systems. Some sufficient conditions for the strong convergence of weighted sum for the function of Markov Chains are obtained. The relevant results will be obtained from the analysis of Theorem 3 and Corollary 3.

Theorem 3. Assume that a Markov chain $\left\{\left(X_{n}, \xi_{n}\right): n \geq 0\right\}$ is defined on $(\Omega, \mathscr{F}, P)$ with values on set of $X \times \Theta$. $\left\{F_{n}\left(X_{n}, \xi_{n}\right): n \geq 0\right\}$ is a sequence of measurable functions defined on $(X \times \Theta, \mathscr{A} \times \mathscr{B})$. $\left\{a_{n}, n \geq 0\right\}$ and $\left\{b_{n}, n \geq 0\right\}$ are arbitrary sequences of positive real numbers, respectively, and we denote $c_{n}=b_{n} / a_{n}, b_{n} \uparrow \infty$, if the following conditions both hold:

(a) $\sum_{m=1}^{\infty} c_{m}^{-1} E\left|F_{m}\left(X_{m}, \xi_{m}\right)\right| I_{\left\{\left|F_{m}\left(X_{m}, \xi_{m}\right)\right|>c_{m}\right\}}<\infty$;

(b) $\sum_{m=1}^{\infty} c_{m}^{-p} E\left|F_{m}\left(X_{m}, \xi_{m}\right)\right|^{p} I_{\left\{\left|F_{m}\left(X_{m}, \xi_{m}\right)\right| \leq c_{m}\right\}}<\infty$, where there is $1 \leq p \leq 2$, 
such that, for any $k \geq 1$, we can get that the following series are convergent almost surely:

$$
\begin{aligned}
& \sum_{m=0}^{\infty} \frac{F_{m}\left(X_{m}, \xi_{m}\right)-E\left(F_{m}\left(X_{m}, \xi_{m}\right) \mid X_{m-k}, \xi_{m-k}\right)}{c_{n}} \\
& \quad \text { a.s.convergence, }
\end{aligned}
$$

and we have

$$
\begin{aligned}
\lim _{n \longrightarrow \infty} & \frac{1}{b_{n}} \sum_{m=0}^{n} a_{m}\left(F_{m}\left(X_{m}, \xi_{m}\right)\right. \\
& \left.-E\left(F_{m}\left(X_{m}, \xi_{m}\right) \mid X_{m-k}, \xi_{m-k}\right)\right)=0 \text { a.s.. }
\end{aligned}
$$

Consequently, we get Corollary 3 as follows.

Corollary 3. Assume that a Markov chain $\left\{\left(X_{n}, \xi_{n}\right): n \geq 0\right\}$ is defined on $(\Omega, \mathscr{F}, P)$ with values on set of $X \times \Theta$. $\left\{F_{n}\left(X_{n}, \xi_{n}\right): n \geq 0\right\}$ is a sequence of measurable functions defined on $(X \times \Theta, \mathscr{A} \times \mathscr{B})$, and it satisfies $\left\{F_{n}\left(X_{n}, \xi_{n}\right)\right\}<V$. $\left\{a_{n}, n \geq 0\right\}$ and $\left\{b_{n}, n \geq 0\right\}$ are arbitrary sequences of positive real numbers, respectively, and we denote $c_{n}=b_{n} / a_{n}, b_{n} \uparrow \infty$. For arbitrary $x>0$, we denote $N(x)=\operatorname{Card}\left\{n: c_{n} \leq x\right\}$, if $V$ satisfies the following two conditions:

(c) $E N(V)<\infty$;

(d) $\int_{1}^{\infty} E N(V / t) d t<\infty$. In addition, one of the following conditions is satisfied:

(e) $\int_{0}^{\infty} t^{p-1} P(V>t) \int_{t}^{\infty} N(y) / y^{p+1} d y d t<\infty$, where there is $1 \leq p \leq 2$;

(f) $\max _{0 \leq j \leq n} c_{j}^{p} \sum_{m=n}^{\infty} c_{m}^{-p}=O(n)$, where there is $1 \leq p \leq 2$;

(g) $\int_{0}^{1} E N\left(V / t^{1 / p}\right) d t<\infty$, where there is $1 \leq p \leq 2$, and, for any $k \geq 1$, both (31) and (32) hold.

Proof of Theorem 3. In a similar way to Theorem 1, we also discuss two situations in the process of proof. One situation is $k=1$; the other situation is $k>1$.

We consider the first situation of $k=1$. For any $m \geq 0$, we denote that

$$
\begin{aligned}
Z_{m} & =\frac{F_{m}\left(X_{m}, \xi_{m}\right) I_{\left\{\left|F_{m}\left(X_{m}, \xi_{m}\right)\right| \leq c_{m}\right\}}}{c_{m}}-\frac{E\left(F_{m}\left(X_{m}, \xi_{m}\right) I_{\left\{\left|F_{m}\left(X_{m}, \xi_{m}\right)\right| \leq c_{m}\right\}} \mid X_{m-1}, \xi_{m-1}\right)}{c_{m}} ; \\
Z_{m}^{\prime} & =\frac{F_{m}\left(X_{m}, \xi_{m}\right) I_{\left\{\left|F_{m}\left(X_{m}, \xi_{m}\right)\right|>c_{m}\right\}}}{c_{m}}
\end{aligned}
$$

Under condition (a), it is known that the following series are absolute convergent almost surely:

$$
\sum_{m=0}^{\infty}\left|Z_{m}^{\prime}\right|<\infty \text { a.s. }
$$

Hence, the following series are convergent almost surely:

$$
\sum_{m=0}^{\infty} Z_{m}^{\prime} \text { a.s.. }
$$

Meanwhile, the following series of conditional expectation are convergent almost surely:

$$
\sum_{m=0}^{\infty} E\left(Z_{m}^{\prime} \mid X_{m-1}, \xi_{m-1}\right) \text { a.s.. }
$$

Then, due to the orthogonal properties of the martingale difference sequence, it is known that, for any $1 \leq p \leq 2$, we can get

$$
E\left|\sum_{m=0}^{n} Z_{m}\right|^{2}=\sum_{m=0}^{n} E Z_{m}^{2} \leq C \sum_{m=0}^{n} \frac{1}{c_{m}^{p}} E\left|F_{m}\left(X_{m}, \xi_{m}\right)\right|^{p} I_{\left\{\left|F_{m}\left(X_{m}, \xi_{m}\right)\right| \leq c_{m}\right\}} .
$$

Through the combination of condition (b) and formula (37), we have the formula $\sup _{n \geq 0} E\left|\sum_{m=0}^{n} Z_{m}\right|^{2}<\infty$. In other words, this formula shows that the result of $\left\{\sum_{m=0}^{n} Z_{m}, \sigma_{n}, n \geq 0\right\}$ is a bounded martingale on $L^{2}$. Thus, we have that the series $\sum_{m=0}^{\infty} Z_{m}$ are convergent almost surely, which is the weighted sum mentioned in the previous section.

The joint two formulas (35) and (36) on almost sure convergence show that formula (31) holds for the situation of $k=1$, and formula (32) for the situation of $k=1$ is also true by Kronecker's Lemma.

Finally, the proof of the other situation of $k>1$ is similar to Theorem 1, so it is omitted.

Here Theorem 3 has been proven regarding the strong convergence of weighted sums. We proved the similar conclusions, but it was from a different viewpoint compared to previous research.

Remark 2. Both Theorem 3 and Theorem 2.1 in literature [25] give the sufficient conditions for strong convergence of weighted sums of Markov chains, which is unlike the preconditions in these two theorems. Furthermore, the results of the strong convergence of weighted sums can be demonstrated under weaker sufficient conditions compared with Theorem 1 in literature [26].

Proof of Corollary 3. Firstly, if $V$ satisfies conditions (c), (d), and (e), for any $k \geq 1$, then both (31) and (32) hold. Now let us show this proof process as follows.

It is only necessary to verify that condition (a) and condition (b) of Theorem 3 are immediately available. As we have the inequality 


$$
\sum_{m=1}^{\infty} P\left(\left|F_{m}\left(X_{m}, \xi_{m}\right)\right|>c_{m}\right) \leq C \sum_{m=1}^{\infty} P\left(V>c_{m}\right) \leq C E N(V)
$$

we can derive the following inequality:

$$
\begin{aligned}
& \sum_{m=1}^{\infty} c_{m}^{-1} E\left|F_{m}\left(X_{m}, \xi_{m}\right)\right| I_{\left\{\left|F_{m}\left(X_{m}, \xi_{m}\right)\right|>c_{m}\right\}} \\
& \quad \leq C \sum_{m=1}^{\infty} \frac{1}{c_{m}} \int_{c_{m}}^{\infty} P\left(\left|F_{m}\left(X_{m}, \xi_{m}\right)\right|>t\right) \mathrm{d} t \\
& \quad \leq C \sum_{m=1}^{\infty} \frac{1}{c_{m}}\left(c_{m} P\left(\left|F_{m}\left(X_{m}, \xi_{m}\right)\right|>c_{m}\right)\right. \\
& \left.\quad+\int_{c_{m}}^{\infty} P\left(\left|F_{m}\left(X_{m}, \xi_{m}\right)\right|>t\right) \mathrm{d} t\right) \\
& \quad \leq C\left(\sum_{m=1}^{\infty} P\left(V>c_{m}\right)+\sum_{m=1}^{\infty} \int_{1}^{\infty} P\left(V>t c_{m}\right) \mathrm{d} t\right) \\
& \quad \leq C\left(E N(V)+\int_{1}^{\infty} E N\left(\frac{V}{t}\right) \mathrm{d} t\right)
\end{aligned}
$$

and, from conditions (c) and (d) of this corollary, we can see that condition (a) of Theorem 3 holds.

By Lemma 2, we have the inequality

$$
\begin{aligned}
& \sum_{m=1}^{\infty} c_{m}^{-p} E\left|F_{m}\left(X_{m}, \xi_{m}\right)\right|^{p} I_{\left\{\left|F_{m}\left(X_{m}, \xi_{m}\right)\right| \leq c_{m}\right\}} \\
& \leq C \sum_{m=1}^{\infty} P\left(V>c_{m}\right)+C \sum_{m=1}^{\infty} c_{m}^{-p} E V^{p} I_{\left\{V \leq c_{m}\right\}} \\
& \quad \leq C E N(V)+C \sum_{m=1}^{\infty} c_{m}^{-p} E V^{p} I_{\left\{V \leq c_{m}\right\}},
\end{aligned}
$$

as well as the inequality

$$
\begin{aligned}
\sum_{m=1}^{\infty} c_{m}^{-p} E|V|^{p} I_{\left\{|V| \leq c_{m}\right\}} & \leq C \sum_{m=1}^{\infty} c_{m}^{-p} \int_{0}^{c_{m}} t^{p-1} P(|V|>t) \mathrm{d} t \\
& =C \int_{0}^{\infty} t^{p-1} P(V>t) \sum_{\left\{m: c_{m}>t\right\}} c_{m}^{-p} \mathrm{~d} t \\
& \leq C \int_{0}^{\infty} t^{p-1} P(V>t) \int_{t}^{\infty} \frac{N(y)}{y^{p+1}} \mathrm{~d} y \mathrm{~d} t .
\end{aligned}
$$
that

The last inequality above is established based on the facts

$$
\begin{gathered}
\sum_{\left\{m: c_{m}>t\right\}} c_{m}^{-p}=\lim _{s \longrightarrow \infty} \sum_{\left\{m: t<c_{m}<s\right\}} c_{m}^{-p}=\lim _{s \longrightarrow \infty} \int_{t}^{s} y^{-p} \mathrm{~d} N(y) \\
=\lim _{s \longrightarrow \infty}\left(s^{-p} N(s)-t^{-p} N(t)+p \int_{t<y \leq s} N \frac{(y)}{y^{p+1}} \mathrm{~d} y\right) ; \\
s^{-p} N(s) \leq p \int_{s}^{\infty} N \frac{(y)}{y^{p+1}} \mathrm{~d} y \longrightarrow 0, \\
s \longrightarrow \infty .
\end{gathered}
$$

Then, by the combination of formula (40), formula (41), condition (c), and condition (e), we know that condition (b) of Theorem 3 holds. Therefore, the proof is completed when conditions (c), (d), and (e) are satisfied.

Secondly, if $V$ satisfies conditions (c), (d), and (f), for any $k \geq 1$, we can get the same conclusions. The proof process is as follows.

Following the proof of Corollary 3 by using formula (40), we only need to prove

$$
\sum_{m=1}^{\infty} c_{m}^{-p} E V^{p} I_{\left\{V \leq c_{m}\right\}}<\infty .
$$

In fact, given some particular values $d_{0}=0$, $d_{n}=\max _{1 \leq m \leq n} c_{m}$, combining the two conditions of (c) and (f), we have 


$$
\begin{aligned}
\sum_{m=1}^{\infty} c_{m}^{-p} E V^{p} I_{\left\{V \leq c_{m}\right\}} & \leq \sum_{m=1}^{\infty} c_{m}^{-p} E V^{p} I_{\left\{V \leq d_{m}\right\}} \\
& =\sum_{m=1}^{\infty} \sum_{j=1}^{m} c_{m}^{-p} E V^{p} I_{\left\{d_{j-1}<V \leq d_{j}\right\}}=\sum_{j=1}^{\infty} E V^{p} I_{\left\{d_{j-1}<V \leq d_{j}\right\}} \sum_{m=j}^{\infty} c_{m}^{-p} \\
& \leq \sum_{j=1}^{\infty} P\left(d_{j-1}<V \leq d_{j}\right) d_{j}^{p} \sum_{m=j}^{\infty} c_{m}^{-p} \leq C \sum_{j=1}^{\infty} j P\left(d_{j-1}<V \leq d_{j}\right) \\
& =C \sum_{j=1}^{\infty} \sum_{m=1}^{j} P\left(d_{j-1}<V \leq d_{j}\right) \leq C \sum_{m=1}^{\infty} \sum_{j=m}^{\infty} P\left(d_{j-1}<V \leq d_{j}\right) \\
& =C \sum_{m=1}^{\infty} P\left(V>d_{m-1}\right) \leq C\left(\sum_{m=1}^{\infty} P\left(V>c_{m}\right)+1\right)<\infty .
\end{aligned}
$$

Therefore, the proof is completed when conditions (c), (d), and (f) are satisfied.

Thirdly, if $V$ satisfies conditions (c), (d), and (g), for any $k \geq 1$, we can also have formulas (31) and (32). The proof process is as follows.

Given $s=m^{p} t$, we use the similar proof method of the first case by condition ( $\mathrm{g}$ ); then we have

$$
\begin{aligned}
\sum_{m=1}^{\infty} c_{m}^{-p} E V^{p} I_{\left\{V \leq c_{m}\right\}} & \leq C \sum_{m=0}^{\infty} \frac{1}{c_{m}^{p}} \int_{0}^{c_{m}^{p}} P\left(V^{p}>s\right) \mathrm{d} s \\
& \leq \int_{0}^{1} E N\left(\frac{V}{t^{1 / p}}\right) \mathrm{d} t<\infty .
\end{aligned}
$$

Therefore, the proof is completed when conditions (c), (d), and (g) are satisfied.

Remark 3. The sufficient conditions of Corollary 3 are similar to those of Theorem 2.1 and Theorem 2.2 in literature [25], which shows that the conditions are applicable to the single infinite environment or double infinite environment.

\section{Conclusions}

From the above discussion, this study confirms that a series of limit theorems for the function of Markov chains in the environment of single infinite Markovian systems are proven. Furthermore, consistent with the literature, the study has found the sufficient conditions for convergence. Moreover, the results of strong law of large numbers and strong convergence of the weighted sums in the infinite environment are given by constructing martingale differential sequence. The finding of preconditions used in this research is broadly different from the previous observations. Although there are similarities between conclusions, they can be expressed by more sufficient conditions of the limit theorems, which are simpler than the analytical techniques proposed earlier. For the further research work, it may be extended to the limit theory related to the function of Markov chain in the environment of double infinite Markovian systems on finite state space.

\section{Data Availability}

No data were used to support this study.

\section{Conflicts of Interest}

The authors declare that there are no conflicts of interest regarding the publication of this paper.

\section{Acknowledgments}

This work was supported by the National Natural Science Foundation of China (NSFC) (no. 71974204) and the "Fundamental Research Funds for the Central Universities," Zhongnan University of Economics and Law (no. 2722020JX005).

\section{References}

[1] R. Cogburn, "Markov chains in random environments: the case of Markovian environments," The Annals of Probability, vol. 8, no. 5, pp. 908-916, 1980.

[2] K. Nawrotzki, "Ergodic theorem for random-processes with embedded homogeneous flow," Theory of Probability and Its Applications, vol. 26, no. 2, pp. 388-392, 1981.

[3] K. Nawrotzki, "Discrete open systems of Markov chains in random environment II," Elektron Informationsverarb. Kybernet, vol. 18, pp. 569-599, 1982.

[4] R. Cogburn, "The ergodic theory of Markov chains in random environments," Zeitschrift für Wahrscheinlichkeitstheorie und Verwandte Gebiete, vol. 66, no. 1, pp. 109-128, 1984.

[5] R. Cogburn, "On direct convergence and periodicity for transition probabilities of Markov chains in random environments," The Annals of Probability, vol. 18, no. 2, pp. 642-654, 1990.

[6] R. Cogburn, "On the central limit theorem for Markov chains in random environments," The Annals of Probability, vol. 19, no. 2, pp. 587-604, 1991.

[7] S. Orey, "Markov chains with stochastically stationary transition probabilities," The Annals of Probability, vol. 19, no. 3, pp. 907-928, 1991.

[8] W. Liu, "Relative entropy densities and a class of limit theorems of seqence of $\mathrm{m}$-valued random variables," The Annals of Probability, vol. 18, no. 2, pp. 829-839, 1990. 
[9] G. X. Liu and W. Liu, "Some strong limit theorems relative to the geometric average of random transition probabilities of arbitrary finite nonhomogeneous Markov chain," Statistics \& Probability Letters, vol. 21, pp. 77-83, 1994.

[10] H. X. Wang and Y. L. Dai, "Poisson's limit law of Markov chain in Markov environment," Journal of Mathematics, vol. 40, pp. 265-270, 1997.

[11] Y. Q. Li, "Some notes on Markov chain in Markov environment," Advances in Mathematics, vol. 28, pp. 358-360, 1999.

[12] D. F. Fang, "The Shannon-McMillan-Breiman theorem of the Markov chain in the Markov environment," Application Probability and Statistics, vol. 16, pp. 295-298, 2000.

[13] D. F. Fang, H. X. Wang, and M. N. Tang, "Possion limit theorem for countable Markov chain in Markovian environments," Applied Mathematics and Mechanics, vol. 24, no. 3, pp. 298-306, 2003.

[14] Y. Q. Li, S. M. Wang, and Y. L. Hu, "A class of strong limit theorem of Markov chain in Markov environment," Advances in Mathematics, vol. 37, pp. 539-550, 2008.

[15] Z. Z. Wang, "A kind of asymptotic properties of moving averages for Markov chains in Markovian environments," Communications in Statistics-Theory and Methods, vol. 46, no. 22, pp. 10926-10940, 2017.

[16] M. L. Guo, "The strong law of large numbers for function of Markov chain in random environment," Application Probability and Statistics, vol. 12, pp. 154-160, 2004.

[17] Y. Q. Li, "Interrelation between Markov chain and Markov double chain in random environment," Journal of Mathematics, vol. 49, pp. 1373-1380, 2006.

[18] C. G. Wan, "The limit theorem of Markov chain function in random environment," Mathematica Applicata, vol. 23, pp. 618-624, 2010.

[19] D. H. Hu and X. Y. Hu, "On Markov chains in space-time random environments," Acta Mathematica Scientia, vol. 29, no. 1, pp. 1-10, 2009.

[20] W. Liu, C. Ma, Y. Li, and S. Wang, "A strong limit theorem for the average of ternary functions of Markov chains in biinfinite random environments," Statistics \& Probability Letters, vol. 100, pp. 12-18, 2015.

[21] Z. Shi, D. Bao, Y. Fan, and B. Wu, "The asymptotic equipartition property of Markov chains in single infinite Markovian environment on countable state space," Stochastics, vol. 91, no. 6, pp. 945-957, 2019.

[22] C. G. Wan, "The strong law of large numbers for function of Markov chain in Markovian environment," Application Probability and Statistics, vol. 19, pp. 1545-2160, 2003.

[23] Y. Q. Li, "The strong law of large numbers for function of Markov chain in Markovian environment with countable state," Journal of Mathematics, vol. 23, pp. 484-490, 2003.

[24] M. L. Guo, "The strong law of large numbers for Markov chain in double infinite environments," Mathematica Applicata, vol. 18, pp. 174-180, 2005.

[25] L. Liu and C. G. Wan, "Strong convergence for weighted sums of Markov chains in bi-infinite environments," Journal of Applied Probability and Statistics, vol. 28, pp. 12-20, 2012.

[26] C. G. Wan, "The strong convergence of weighted sums of function for finite state Markov chains in single infinitely Markovian environments," Journal of Applied Probability and Statistics, vol. 29, pp. 633-641, 2013.

[27] Y. Yang and K. C. Yuen, "Finite-time and infinite-time ruin probabilities in a two-dimensional delayed renewal risk model with Sarmanov dependent claims," Journal of Mathematical Analysis and Applications, vol. 442, no. 2, pp. 600-626, 2016.
[28] Y. Yang, W. Su, and Z. Zhang, "Estimating the discounted density of the deficit at ruin by Fourier cosine series expansion," Statistics \& Probability Letters, vol. 146, pp. 147155, 2019.

[29] W. Yu, Y. Yong, G. Guan, Y. Huang, W. Su, and C. Cui, "Valuing guaranteed minimum death benefits by cosine series expansion," Mathematics, vol. 7, no. 9, p. 835, 2019.

[30] Z. M. Zhang, Y. D. Yong, and W. G. Yu, "Valuing equitylinked death benefits in general exponential Lévy models," Journal of Computational and Applied Mathematics, vol. 365, Article ID 112377, 2020.

[31] Y. Yang, T. Jiang, K. Wang, and K. C. Yuen, "Interplay of financial and insurance risks in dependent discrete-time risk models," Statistics and Probability Letters, vol. 162, Article ID 108752, 2020.

[32] W. Yu, P. Guo, Q. Wang et al., "On a periodic capital injection and barrier dividend strategy in the compound Poisson risk model," Mathematics, vol. 8, no. 4, p. 511, 2020.

[33] W. G. Yu, F. Wang, Y. J. Huang, and H. D. Liu, "Social optimal mean field control problem for population growth model," Asian Journal of Control, pp. 1-8, 2019.

[34] Y. L. Wu and X. T. Wu, "The strong law of large numbers for function of Markov chain in Markovian environment," Journal of Mathematics, vol. 27, pp. 579-586, 2013. 